\title{
5-year benefits of rituximab for ABO-mismatched renal grafts
}

Clinical uses of the anti-CD20 monoclonal antibody rituximab extend to a wide variety of hematological and autoimmune disorders. According to results from a new study conducted in Japan, rituximab also improves the outcomes of patients with ABOincompatible kidney grafts. “...we found that the rituximab-containing preconditioning regimen achieved ... effects that far exceeded our initial expectation," write the authors.

Fuchinoue and co-workers assessed the clinical outcomes of 408 patients with end-stage renal disease who had received kidney allografts from spouses or living related donors between January 2002 and December 2008. The patients were grouped according to their ABO graft compatibility status and whether rituximab had been used in addition to the standard preoperative immunosuppression induction regimen. The groups were as follows: 280 patients who underwent ABO-compatible kidney transplantation (control group), 63 patients who underwent $\mathrm{ABO}$ incompatible kidney transplantation without rituximab induction but with splenectomy during transplantatation,

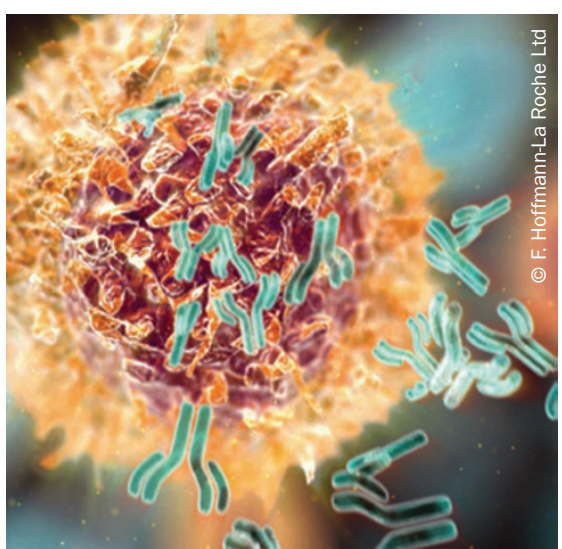

and 50 patients who underwent $\mathrm{ABO}$ incompatible kidney transplantation with rituximab. Patients who underwent $\mathrm{ABO}$-incompatible transplantation were significantly older than controls and were more likely to have received grafts from spouses. In Japan, the researchers explain, parents (who share common ABO loci with their children) often act as donors for younger patients, whereas for older patients, spouses are usually the most frequent donors. Donor and graft characteristics were similar for all three groups.

The researchers found that patients who received rituximab tended to have better outcomes and graft survival than did patients in the other two groups, although the differences were largely not statistically significant. 5-year graft survival was $100 \%$ in rituximab-treated patients, compared with $90.3 \%$ in patients with $\mathrm{ABO}$-incompatible grafts who did not receive rituximab and $88.4 \%$ in controls. Episodes of acute rejection tended to be fewer and milder in the rituximab-treated group than in the other two groups. Importantly, overall infection rates as well as cytomegalovirus infection rates (a serious complication after transplantation) in rituximab-treated patients were similar to those in the other two groups.

"...in the long term," the researchers' report concludes, "a preoperative regimen including rituximab ... yields a better outcome than [regimens without rituximab], without any increase in the risk of infection."

Steven E. Bradshaw

Original article Fuchinoue, S. et al. The 5-year outcome of ABO-incompatible kidney transplantation with rituximab induction. Transplantation doi:10.1097/ TP.0b013e31820f08e8 